\title{
AVALIAÇÃO DA FLEXIBILIDADE E FORÇA MUSCULAR DE MEMBROS INFERIORES EM IDOSAS PRATICANTES DE HIDROGINÁSTICA
}

\section{Daniel Vicentini de Oliveira}

Doutorando em Gerontologia pela Faculdade de Ciências Médicas da Universidade Estadual de Campinas (UNICAMP), Brasil.

E-mail: d.vicentini@hotmail.com

Amanda Piva Pereira

Graduada em Educação física pela Faculdade Metropolitana de Maringá (FAMMA), Brasil.

José Roberto Andrade do Nascimento Júnior

Doutor em Educação física (UEM); Docente adjunto no departamento de Educação física da Universidade Federal do Vale do São Francisco (UNIVASF), Brasil.

\section{Mateus Dias Antunes}

Mestrando no Programa de Pós-graduação em Promoção da Saúde (PPGPS) do Centro Universitário de Maringá (UNICESUMAR), Bolsista Capes, Brasil.
RESUMO: Este estudo teve como objetivo avaliar a flexibilidade e força muscular de membros inferiores de idosas praticantes de hidroginástica no município de Sarandi, Estado do Paraná. Foram avaliadas 31 idosas praticantes de hidroginástica. Para a avaliação da flexibilidade foi utilizado o teste de Sentar e Alcançar e para a avaliação de força muscular de membros inferiores foi utilizado o teste de sentar e levantar de cadeira em 30 segundos. A análise de dados foi realizada por meio da estatística descritiva e inferencial.

PALAVRAS CHAVE: Aptidão física; Envelhecimento; Exercício físico.

\section{FLEXIBILITY AND MUSCLE STRENGTH OF THE LOWER MEMBERS IN ELDERLY PEOPLE PRACTICING HYDROGYMNASTICS}

ABSTRACT: Flexibility and muscle strentgh of the lower body members of elderly people who practice hydrogymnastics in Sarandi PR Brazil, are assessed. Thirty-one elderly people were evaluated. Flexibility was assessed by the Sit and Reach test, while the muscle strentgh of the lower members was evaluated by the Sit and Raise the Chair in 30 seconds. Data analysis was done by descriptive and inferential statistics.

KEY WORDS: Physical capacity; Aging; Physical exercises.

\section{INTRODUÇÃO}

O processo de envelhecimnto acarreta um declínio das funções biológicas, intelectuais, funcionais e sociais (ZAMBON et al., 2016). Segundo Coelho et al. (2013), este processo é caracterizado por alterações que podem dificultar as condições de vida dos idosos. Além disso, ocorrem alterações nos componentes da capacidade funcional, como perda da elasticidade e viço da pele; diminuição da força muscular, da agilidade e da mobilidade das articulações (FIDELIS; PATRIZZI; WALSH, 2013), que correspondem as atividades básicas de vida diária (ABDVs), como levantar-se da cadeira, caminhar, subir e descer escadas, e atividades instrumentais de vida diária (AVIDs) (FLECK; KRAEMER, 2006).

Dentre estas alterações, podemos citar a diminuição da flexibilidade (FL) e força muscular (FM), que são aspectos da aptidão física relacionada a saúde e altamente requisitadas nas Atividades de 
Vida Diária (AVDs) (FIDELIS; PATRIZZI; WALSH, 2013). A FL se torna necessária não só na realização das AVDs mas também em atividades circunstanciais especificas, por exemplo, na prevenção de quedas (ALBINO et al., 2012). Para Arena (2009), a boa FL é essencial para o bom desempenho físico, tanto em uma atividade física estruturada como nas AVDs.

A diminuição dos níveis de FM podem promover dependência das AVDs, promovendo a perda de massa muscular nos membros inferiores (BATISTA et al., 2014). Nota-se que este processo de perda de massa muscular ocorre primeiro e em maior grau nos membros inferiores (MMII), uma vez que os idosos tendem a passar mais tempo sentados, solicitando mais os membros superiores (MMSS) (ROMA et al., 2013).

Como fator preventivo e de controle destas alterações, o exercício físico vem a contribuir para a conservação ou até mesmo melhora das alterações abordadas acima, pois ao se desenvolver um determinado tipo de exercício físico, devemos levar em consideração a necessidade do mesmo, assim procurando sempre a facilitar a execução das AVDs de idosos (PLUMMER et al., 2016; CHAN et al., 2015; ZHANG; SHUAI, 2015; KARR et al., 2014; GINÉ-GARRICA et al., 2014; VRIES et al., 2012).

Segundo Coelho et al. (2013), não tem como adotar um método único de prescrição de exercício, mas existem algumas etapas que auxiliam na prescrição, como o diagnóstico, o prognóstico, a programação, a implementação e a avaliação. Essas etapas quando bem aplicadas levam um programa de exercício ao resultado positivo. O exercício físico para idosos deve contemplar atividades que ajudem no desempenho das AVDs do idoso.

Uma das modalidades de exercício físico mais procurada pelos idosos é a hidroginástica, pois segundo Ferreira (2007), a mesma facilita os movimentos, tornando a atividade mais agradável, segura e dinâmica, além de serem excelentes para a manutenção das articulações e músculos. A hidroginástica é um programa de exercícios aquáticos específicos, que segundo Lucchesi (2013), é composta de movimentos rítmicos, coreografados ou não, utilizando-se da água com suas propriedades como recurso principal para oferecer resistência e sobrecarga natural aos movimentos, além de ser uma atividade contagiante e divertida.
Segundo Aboarrage (2008), assim como qualquer outra forma de exercitação, os exercícios de hidroginástica estão voltados ao alcance de objetivos pré-determinados, de modo que o praticante adquira boa condição de saúde ou mantenha a já adquirida, além de aumentar a sua independência, visto que para um idoso o fato de não depender da ajuda de ninguém para realizar as suas AVDs e AIVDs é considerado como uma boa condição de saúde e para que consiga realizar as mesmas se torna necessária a boa capacidade física dentre estas FL e FM.

Posto isso, o presente estudo teve como objetivo avaliar a flexibilidade e força muscular de membros inferiores de idosas praticantes de hidroginástica, buscando especificamente verificar a correlação entre o tempo de prática, a flexibilidade e a força muscular de membros inferiores das idosas.

\section{MÉTODOS}

Trata-se de um estudo descritivo, observacional e transversal, realizado entre fevereiro a agosto de 2015 , aprovado pelo Comitê de Ética e Pesquisa do Centro Universitário Cesumar (UniCesumar), por meio do protocolo 1.173.073/2015.

A amostra foi escolhida de forma intencional e por conveniência, e foi composta por 31 mulheres. Foram incluídas todas as mulheres com idade igual ou superior a 60 anos até no máximo 80 anos, praticantes de hidroginástica há pelo menos três meses e no mínimo duas vezes semanais, na única academia que oferecia esta modalidade de exercício físico no município de Sarandi. Foram excluídas as idosas praticantes de outra (s) modalidade (s) de exercício físico, além da hidroginástica; idosas que apresentassem alterações neurológicas e ortopédicas que impedissem a realização do teste e aplicação do questionário, assim como idosas com mais de 80 anos e idosos do sexo masculino, pela baixa prevalência de ambos na prática de hidroginástica.

Foi utilizado um questionário semiestruturado, composto por informações referentes à idade, ao estado civil (casada ou não casada), à escolaridade, à situação ocupacional, à renda mensal em salário mínimo (SM) de referência no Censo Demográfico 2015 - IBGE, 
à quantidade de medicamentos utilizados, à queda nos últimos seis meses, ao motivo para a prática de hidroginástica, à frequência semanal e ao tempo de prática da hidroginástica. No item estado civil, para fins de análise estatística, foram consideradas não casadas também as idosas viúvas, separadas ou divorciadas.

Para a avaliação da FL foi utilizado o teste de sentar e alcançar proposto por Wells e Dillon (1956) e validado, no Brasil, por Araújo (1999), que é considerado de fácil aplicação e baixo custo operacional e recomendado e utilizado pelas principais baterias de testes já padronizadas em todo o mundo, e tem como objetivo principal avaliar a FL dos músculos isquiotibiais.
O teste é realizado utilizando uma caixa medindo $30,5 \mathrm{~cm} \times 30,5 \mathrm{~cm} \times 30,5 \mathrm{~cm}$ com uma escala de $26,0 \mathrm{~cm}$ em seu prolongamento, sendo que o ponto 0 se encontra na extremidade mais próxima do avaliado e o $26 \mathrm{~cm}$ coincide com o ponto de apoio dos pés. Para a realização do teste o participante senta-se com as pernas juntas, os joelhos estendidos e as plantas dos pés colocadas contra a borda da caixa. O participante tenta alcançar lentamente à frente o mais distante possível ao longo do topo do banco, conservando as duas mãos paralelas, não podendo flexionar os joelhos, deverá manter esta posição momentaneamente para a avaliação (HEYWARD, 2004). Os valores de referência estão abordados na Tabela 1.

Tabela 1. Classificação do teste de flexibilidade para mulheres

\begin{tabular}{c|c|c|c|c|c|c}
\hline $\begin{array}{c}\text { Classificação sentar e alcançar } \\
\text { mulheres }\end{array}$ & $\begin{array}{c}\mathbf{1 5} \text { a } \mathbf{1 9} \\
\text { anos de } \\
\text { idade }\end{array}$ & $\begin{array}{c}\mathbf{2 0} \text { a } \mathbf{2 9} \\
\text { anos de } \\
\text { idade }\end{array}$ & $\begin{array}{c}\mathbf{3 0} \text { a 39 } \\
\text { anos de } \\
\text { idade }\end{array}$ & $\begin{array}{c}\mathbf{4 0} \text { a } 49 \\
\text { anos de } \\
\text { idade }\end{array}$ & $\begin{array}{c}\mathbf{5 0} \text { a 59 } \\
\text { anos de } \\
\text { idade }\end{array}$ & $\begin{array}{c}60 \text { a } 69 \\
\text { anos de } \\
\text { idade }\end{array}$ \\
\hline Excelente & $\geq 43$ & $\geq 41$ & $\geq 41$ & $\geq 38$ & 39 & $\geq 35$ \\
\hline Acima da média & $38-42$ & $37-40$ & $36-40$ & $34-37$ & $33-38$ & $31-34$ \\
\hline Média & $34-37$ & $33-36$ & $32-35$ & $30-33$ & $30-32$ & $27-30$ \\
\hline Abaixo da média & $29-33$ & $28-32$ & $27-31$ & $25-29$ & $25-29$ & $23-26$ \\
\hline Ruim & $\leq 28$ & $\leq 27$ & $\leq 26$ & $\leq 24$ & $\leq 24$ & $\leq 22$ \\
\hline
\end{tabular}

Para a avaliação de FM em membros inferiores foi utilizado o teste de sentar e levantar de cadeira em 30 segundos, proposto por Rikli e Jones (2008). Para a realização do mesmo se fez necessária uma cadeira com o encosto reto, com altura do assento de $43 \mathrm{~cm}$ e um cronômetro. $\mathrm{O}$ teste ocorre de seguinte forma: o cliente é instruído a sentar-se na cadeira com os braços cruzados na altura do peito de forma que formará um "X", ao sinal do avaliador o participante deverá levantar, ficar em posição ereta e depois retornar à posição inicial, sendo realizado o máximo de repetições em 30 segundos. Os valores de referência estão abordados na Tabela 2 .

Tabela 2. Classificação do teste de força muscular de membros inferiores em mulheres

\begin{tabular}{c|c|c|c|c|c|c|c}
\hline $\begin{array}{c}\text { Classificação Levantar } \\
\text { e Sentar da cadeira } \\
\text { mulheres }\end{array}$ & $\begin{array}{c}60 \text { a 64 } \\
\text { anos de } \\
\text { idade }\end{array}$ & $\begin{array}{c}\mathbf{6 5} \text { a 69 } \\
\text { anos de } \\
\text { idade }\end{array}$ & $\begin{array}{c}70 \text { a } 74 \\
\text { anos de } \\
\text { idade }\end{array}$ & $\begin{array}{c}75 \text { a 79 } \\
\text { anos de } \\
\text { idade }\end{array}$ & $\begin{array}{c}\mathbf{8 0} \text { a } 84 \\
\text { anos de } \\
\text { idade }\end{array}$ & $\begin{array}{c}\mathbf{8 5} \text { a 89 } \\
\text { anos de } \\
\text { idade }\end{array}$ & $\begin{array}{c}90 \text { a 94 } \\
\text { anos de } \\
\text { idade }\end{array}$ \\
\hline Muito fraco & $\leq 12$ & $\leq 12$ & $\leq 11$ & $\leq 10$ & $\leq 10$ & $\leq 9$ & $\leq 8$ \\
\hline Fraco & $13-15$ & $13-14$ & $12-13$ & $11-13$ & $11-12$ & $10-11$ & $9-10$ \\
\hline Regular & $16-17$ & $15-16$ & $14-16$ & $13-15$ & $13-14$ & $12-13$ & $11-12$ \\
\hline Bom & $18-20$ & $17-19$ & $17-18$ & $16-18$ & $15-16$ & $15-15$ & $12-15$ \\
\hline Muito Bom & $\geq 21$ & $\geq 20$ & $\geq 19$ & $\geq 19$ & $\geq 17$ & $\geq 16$ & $\geq 15$ \\
\hline
\end{tabular}

Fonte: Rikli e Jones (1999). 
O questionário sociodemográfico e de perfil foi aplicado por meio de entrevista para que fossem evitados possíveis erros de interpretações. A coleta de dados foi realizada na própria academia, antes do horário da aula.

Para análise dos dados, foi utilizado frequência e percentual para as variáveis categóricas. Para as variáveis numéricas, inicialmente foi verificada a normalidade dos dados por meio do teste Shapiro-Wilk. Como os dados não apresentaram distribuição normal, foram utilizadas Mediana (Md) e Quartis (Q1; Q3) para a caracterização dos resultados. Na comparação da quantidade de doenças, medicamentos, nível de FL e FMMII em função do tempo de prática (menos de 01 ano e mais de 01 ano), foi utilizado o teste "U" de Mann-Whitney. A correlação entre as variáveis foi feita por meio da correlação de Spearman, sendo adotada a significância de $p<0,05$.

\section{RESULTADOS}

Os dados relacionados ao perfil sociodemográfico e característica pessoais das idosas estão apresentados na Tabela 3.

Tabela 3. Distribuição de frequência do perfil sóciodemográfico das idosas praticantes de hidroginástica

\begin{tabular}{|c|c|c|}
\hline VARIÁVEIS & $f$ & $\%$ \\
\hline \multicolumn{3}{|l|}{ Estado civil } \\
\hline Casada & 22 & 71 \\
\hline Não casada & 09 & 29 \\
\hline \multicolumn{3}{|l|}{ Situação ocupacional } \\
\hline Ativo & 09 & 29 \\
\hline Inativo & 22 & 71 \\
\hline \multicolumn{3}{|l|}{ Renda mensal } \\
\hline 1 a 2 salários mínimos & 24 & 77 \\
\hline Mais de 2 salários mínimos & 07 & 23 \\
\hline \multicolumn{3}{|l|}{ Escolaridade } \\
\hline 1 a 4 anos & 17 & 54 \\
\hline 5 a 8 anos & 7 & 23 \\
\hline Mais de 8 anos & 7 & 23 \\
\hline \multicolumn{3}{|l|}{ Queda nos últimos 6 meses } \\
\hline Sim & 05 & 16 \\
\hline Não & 26 & 84 \\
\hline
\end{tabular}

$f$ : frequência.

Em relação ao perfil de atividade física das idosas (Tabela 2), verificou-se que a maioria das mulheres praticava a hidroginástica entre três meses a um ano (58\%) e que começaram a realizar a mesma por recomendação médica (81\%). 
Tabela 4. Distribuição de frequência do perfil de atividade física das idosas praticantes de hidroginástica

\begin{tabular}{|c|c|c|}
\hline VARIÁVEIS & $f$ & $\%$ \\
\hline \multicolumn{3}{|l|}{ Tempo de prática } \\
\hline Menos de um ano & 18 & 58 \\
\hline Mais de um ano & 13 & 42 \\
\hline \multicolumn{3}{|l|}{ Motivo para a prática } \\
\hline Recomendação médica & 25 & 81 \\
\hline Vontade própria & 06 & 19 \\
\hline \multicolumn{3}{|l|}{ Flexibilidade } \\
\hline Ruim & 22 & 71 \\
\hline Abaixo da média & 05 & 16 \\
\hline Na média/acima da média & 04 & 13 \\
\hline \multicolumn{3}{|l|}{ Força Muscular } \\
\hline Fraco/muito fraco & 03 & 10 \\
\hline Regular & 05 & 16 \\
\hline Bom & 09 & 29 \\
\hline Muito bom & 14 & 45 \\
\hline
\end{tabular}

Não houve diferença significativa $(p>0,05)$ ao comparar a quantidade de medicamentos utilizados, as doenças presentes, o nível de flexibilidade e a força muscular de membros inferiores em função do tempo de prática de hidroginástica (Tabela 3).

Tabela 5. Comparação da quantidade de medicamentos utilizados, doenças presentes, flexibilidade e força muscular das idosas praticantes de hidroginástica em função do tempo de prática

\begin{tabular}{|c|c|c|c|}
\hline \multirow{3}{*}{ VARIÁVEIS } & \multicolumn{2}{|c|}{ Tempo de Prática } & \multirow{3}{*}{$p$} \\
\hline & $\begin{array}{c}3 \text { meses a } 1 \text { ano } \\
\quad(n=18)\end{array}$ & $\begin{array}{l}\text { Mais de } 1 \text { ano } \\
\quad(\mathrm{n}=13)\end{array}$ & \\
\hline & Md (Q1;Q3) & Md (Q1;Q3) & \\
\hline Medicamentos & $2,50(1,00 ; 3,00)$ & $2,00(1,00 ; 3,00)$ & 0,441 \\
\hline Doenças & $2,00(0,75 ; 2,00)$ & $2,00(1,00 ; 2,00)$ & 0,465 \\
\hline Flexibilidade & $20,00(14,75 ; 24,25)$ & $17,00(14,00 ; 21,00)$ & 0,567 \\
\hline Forca Muscular MI & $15,00(12,00 ; 17,25)$ & $15,00(12,50 ; 17,00)$ & 0,953 \\
\hline
\end{tabular}

* Diferença significativa: $p<0,05$ - Teste "U" de Mann-Whitney. MI: membro inferior. Md: mediana, 1;Q3: quartis.

Verificou-se correlação positiva e fraca $(r<0,40)$ entre o nível de flexibilidade e força muscular de membros inferiores das idosas praticantes de hidroginástica $(r=0,271)$, entretanto, não significativa $(p>0,05)$. 


\section{DISCUSSÃO}

Nota-se a prevalência de idosas casadas (71\%), com situação ocupacional inativa (71\%), renda mensal de um a dois salários mínimos (77\%), tempo de escolaridade de um a quatro anos (54\%) e que não sofreram quedas nos últimos seis meses (84\%).

Com o resultado obtido sobre a situação ocupacional das idosas praticantes de hidroginástica, notou-se que $71 \%$ são inativas o que apresenta procura maior pela qualidade de vida e manutenção da saúde apenas por indivíduos inativos o que pode caracterizar falta de tempo para o cuidado com a saúde caso o indivíduo ainda seja ativo. De acordo com Schneider e Irigaray (2008), para muitos idosos, a aposentadoria é um quadro complexo com vários pontos de vista, pois o mesmo pode ser considerado um período de desengajamento da vida social e para outros o início de uma vida social prazerosa onde se inicia a busca por lazer e atividade física.

Analisando a escolaridade das idosas, nota-se que $54 \%$ das mesmas apresentam escolaridade baixa (de 1 a 4 anos). Durante a coleta do questionário, algumas idosas justificaram o nível baixo de escolaridade pelo fato que tinham que trabalhar desde pequenas na lavoura para ajudar na renda da família. O resultado da baixa escolaridade pode ter refletido no resultado encontrado da renda mensal das idosas, haja vista que $77 \%$ ganham de um a dois salários mínimos, pois o indivíduo com menor grau de escolaridade não dispõe de uma gama de serviço que o remunere bem ao logo da sua vida.

Para França e Soares (2009), a perspectiva de crescimento da população idosa aposentada é preocupante, pois o recurso da aposentadoria fornecido para os mesmos não será suficiente para garantir saúde e bem-estar a essa população. Luz et al. (2014) apresentaram resultados similares com os encontrados no presente trabalho em que os idosos detinham grau de escolaridade baixo e renda mensal de um a dois salários mínimos, provenientes da aposentadoria; os autores ainda explicam que o nível baixo de escolaridade interfere na qualidade de vida dos mesmos, pois não são capazes de administrar com qualidade os remédios, a dieta e entre outros.

De acordo com Maciel (2010), um dos motivos da prática de exercício físico pelos idosos serem iniciada somente após a indicação médica, acontece pela deficiência que existe nos programas públicos de incentivo à prática de exercício físico no Brasil. Assim, os idosos procuram a prática somente após um diagnóstico do médico sobre a existência de uma doença, caso houvesse maior investimento do poder público na prática de atividade física, como propagandas informativas, folhetos informativos, palestras de fácil acesso e até mesmo atividades gratuitas esse número poderia ser revertido, pois a busca pela saúde estaria sendo conquistada antes mesmo da doença se manifestar.

Verificou-se a prevalência de mulheres com nível ruim de FL (71\%). Esse valor pode ser explicado pela frequência e pelo volume da prática durante a hidroginástica, sendo esse passível de melhora caso seja apresentada uma ênfase maior de trabalho. Para Almeida, Veras e Doimo (2010), ao final da aula de hidroginástica, deve-se realizar relaxamento, pois nesse momento poderia ser trabalhado com maior intensidade a FL dos alunos envolvidos.

Estudo realizado por Passos et al. (2008) apresenta resultados parecidos aos obtidos no trabalho em questão, em que foram avaliados dois grupos que não apresentaram ganho significativo de FL ao realizar o programa de hidroginástica. O mesmo também justifica os resultados encontrados pela falta de um programa específico que trabalhe a FL durante a aula de hidroginástica, além da intensidade e da sessão não serem o suficiente para resultados significativos.

Assis et al. (2007) apontam que para existir um ganho de FL na hidroginástica basta estender os músculos de 30 a 60 segundos, pois a água irá diminuir o efeito de gravidade nas articulações o que faz com que os movimentos se estendam mais sem ocorrer aumento de pressão nas articulações. Porém, deve ser levado em consideração que grande parte das idosas que procuram um programa de hidroginástica dispõe de algum problema ortopédico, em grande maioria localizado na região do joelho, o que poderia ser um dos agravantes que contribuiram para o baixo nível de FL das idosas estudadas. Os autores supracitados ainda enfatizam que a água ajuda no relaxamento das articulações facilitando o desenvolver do alongamento, porém esse relaxamento apresenta de uma forma tão insignificante e passa a contribuir para o resultado de baixa FL das idosas. 
Os resultados apresentaram idosas com nível muito bom de FM de membros inferiores (45\%), porém esse número poderia ter sido maior caso, durante a realização das aulas, fossem utilizados materiais que oferecem resistência ao membro trabalhado. Utilização de equipamentos resistivos em treinamentos específicos de força na hidroginástica demonstra que podem aumentar a atividade muscular de determinados grupos musculares refletindo em melhores resultados (CARDOSO; MAZO; BALBÉ, 2010).

Segundo Lacourt e Marini (2006), a diminuição da FM também pode estar associada a doenças agudas ou crônicas, hospitalizações por traumas ou por cirurgias, a inatividade física, estes fatores podem explicar porque $10 \%$ da amostra deste estudo apresentam um nível de força muscular fraca/muito fraca.

A manutenção dessa FM é sinal de que os exercícios físicos estão sendo realizados de forma eficaz (FIDELIS; PATRIZZI; WALSH, 2013). Assim pode-se dizer que os escores obtidos em FM, fraca e regular, advém de uma prática de exercício de baixa qualidade; essa prática de baixa qualidade vai implicar diretamente na baixa manutenção da perda de FM e, consequentemente, essa não manutenção acarretará ao idoso uma inatividade maior, assim o idoso passa a ficar mais dependente de ajuda para realizar suas AVDs e AIVDs.

Não houve diferença significativa $(p>0,05)$ ao comparar a quantidade de medicamentos utilizados, doenças presentes, nível de FL e FM de membros inferiores em função do tempo de prática de hidroginástica (Tabela $3)$.

Segundo Lucchetti et al. (2010), a polifarmácia é caracterizada pela administração de cinco ou mais medicamentos por paciente, assim ao analisar os resultados obtidos nota-se que a média de medicamento entre as idosas é de dois a três o que não caracteriza polifarmácia. Um dos motivos que influencia a não utilização de vários medicamentos é a prática de exercício físico, tendo em vista que a análise foi realizada apenas com praticantes de hidroginástica. Supõe-se que a prática de hidroginástica implica na quantidade de medicamentos que a idosa faz uso, pois quanto maior sua inatividade maior será o índice de doenças e, consequentemente, o uso de medicamentos.
Verificou-se correlação positiva e fraca $(r<0,40)$ entre o nível de flexibilidade e força muscular de membros inferiores das idosas praticantes de hidroginástica $(r=$ $0,271)$, entretanto, não significativa $p>0,05$ ).

$O$ estudo apresenta limitações como a presença de uma única academia no município que ofereça hidroginástica para idosas, a ausência de um grupo composto de idosas praticantes de outra (s) modalidade (s) de exercício físico, e de um grupo controle composto por idosas não praticantes de exercícios, para fins de comparação de resultados.

Entretanto, mesmo com as limitações citadas acima, acredita-se que o presente estudo pode oferecer aos profissionais de educação física e saúde importantes conhecimentos sobre a importância de se incentivar a prática de exercícios físicos, como um dos componentes importantes e determinantes da promoção da saúde do idoso.

\section{CONCLUSÃO}

Conclui-se que as idosas praticantes de hidroginástica no município de Sarandi, Paraná possuem classificação muito ruim de FL, avaliada por meio do teste sentar e alcançar, assim como fraca FMI, avaliada pelo teste sentar e levantar da cadeira. Estas conclusões apontam a necessidade de intensificar o trabalho destes componentes físicos nas aulas de hidroginástica.

\section{REFERÊNCIAS}

ABOARRAGE, N. Treinamento de força na água: uma estratégia de observação e abordagem pedagógica. São Paulo: Phorte, 2008.

ALBINO, I. L. R. et al. Influência do treinamento de força muscular e de flexibilidade articular sobre o equilíbrio corporal em idosas. Rev. Bras. Geriatr. Gerontol., v. 15, n. 1, p. 17-25, 2012.

ALMEIDA, A.P.P.V.; VERAS, R.P.; DOIMO, L.A. Avaliação do equilíbrio estático e dinâmico de idosas praticantes de hidroginástica e ginástica. Rev. Bras. Cineantropom. 
Desempenho Hum., v.12, n.1, p. 55- 61, 2010.

ARAÚJO, C.G.S. Teste de sentar-levantar - apresentação preliminar de um procedimento para avaliação em Medicina do Exercício e do Esporte. Rev. Bras. Med. Esporte, v. 5, n. 1, p. 179-182, 1999.

ARENA, S.S. Exercício físico e qualidade de vida: avaliação, prescrição e planejamento. São Paulo: Phorte, 2009.

ASSIS, R.S. et al. A hidroginástica melhora o condicionamento físico dos idosos. RBPFEX, v.1, n.5, p. $62-75,2007$.

BATISTA, F. S. et al. Relationship between lower-limb muscle strength and functional independence among elderly people according to frailty criteria: a crosssectional study. Sao Paulo Med J, v. 132, n. 5, p. 282289, 2014.

CARDOSO, A.S.; MAZO, G.Z.; BALBÉ, G.P. Níveis de força em mulheres idosas praticantes de hidroginástica: um estudo de dois anos. Motriz, v. 16, n. 1, p. 86- 94, 2010.

CHAN, W. C. et al. Efficacy of physical exercise in preventing falls in older adults with cognitive impairment: a systematic review and meta-analysis. J Am Med Dir Assoc, v. 16, n. 2, p. 149-154, 2015.

COELHO, F.G.M. et al. Exercício físico no envelhecimento saudável e patológico: da teoria a pratica. Curitiba: CRV, 2013.

FERREIRA, V. Atividade física na terceira idade: 0 segredo da longevidade. 2. ed. Rio de Janeiro: Sprint, 2007.

FIDELIS, L.T.; PATRIZZI, L.J.; WALSH, I.A.P. Influência da prática de exercícios físicos sobre a flexibilidade, força muscular manual e mobilidade funcional em idosos. Rev. Bras. Geriatr. Gerontol., v.16, n.1, p. 109-116, 2013.

FLECK, S.J.; KRAEMER, W.J. Fundamentos do treinamento de força muscular. Porto Alegre: Artmed, 2006.
FRANÇA, L.H.F.P.; SOARES, D.H.P. Preparação para a aposentadoria como parte da educação ao longo da vida. Psicol., Ciênc. Prof., v.29, n.4, p. 738-751, 2009.

GINÉ-GARRIGA, M. et al. Physical exercise interventions for improving performance-based measures of physical function in community-dwelling, frail older adults: a systematic review and meta-analysis. Arch Phys Med Rehabil, v. 95, n. 4, p. 753-769, 2014.

HEYWARD, V.H. Avaliação física e prescrição de exercícios: técnicas avançadas. São Paulo: Artmed. 2004.

KARR, J. E. et al. An empirical comparison of the therapeutic benefits of physical exercise and cognitive training on the executive functions of older adults: A meta-analysis of controlled trials. Neuropsychology, v. 28, n. 6, p. 829-843, 2014.

LACOURT, M.X.; MARINI, L.L. Decréscimo da função muscular decorrente do envelhecimento e a influência na qualidade de vida do idoso: uma revisão de literatura. RBCEH, Rev. Bras. Ciênc. Envelhecimento Hum., , v.3, n .1, p. 114-121, 2006.

LUCCHESI, G.A. Hidroginástica: aprendendo a ensinar. São Paulo: Cone, 2013.

LUCCHETTI, G. et al. Fatores associados à polifarmácia em idosos institucionalizados. Rev. Bras. Geriatr. Gerontol, v.13, n.1, p. 51-58, 2010.

LUZ, E.P. et al. Perfil sociodemográfico e de hábitos de vida da população idosa de um município da região norte do Rio Grande do Sul, brasil. Rev. Bras. Geriatr. Gerontol, v.17, n.2, p.303-314, 2014.

MACIEL, M.G. Atividade física e funcionalidade do idoso. Motriz, v.16, n.4, p.1024-1032, 2010.

PASSOS, B.M.A. et al. Contribuições da hidroginástica nas atividades da vida diária e na flexibilidade de mulheres idosas. J. Phys. Educ., , v.19, n.1, p. 71- 76, 2008.

PLUMMER, P. et al. Effects of physical exercise interventions on gait-related dual-task interference in older adults: a systematic review and meta-analysis. 
Gerontology, v. 62, n. 1, p. 94-117, 2016.

RIKLI, R.E.; JONES, C.J. Teste de aptidão física para idosos. Barueri: [s.n.], 2008.

ROMA, M. F. B. et al. Effects of resistance training and aerobic exercise in elderly people concerning physical fitness and ability: a prospective clinical trial. Einstei, v. 11, n. 2, p. 153-157, 2013.

SCHNEIDER, R.H.; IRIGARAY, T.Q. O envelhecimento na atualidade: aspectos cronológicos, biológicos, psicológicos e sociais. Estud Psicol., v.25, n.4, p. 585593, 2008.

VRIES, N. M. et al. Effects of physical exercise therapy on mobility, physical functioning, physical activity and quality of life in community-dwelling older adults with impaired mobility, physical disability and/or multimorbidity: a meta-analysis. Ageing res rev., v. 11, n. 1, p. 136-149, 2012.

WELLS, K. F.; DILLON, E. K. The sit and reach: a test of back and leg flexibility. Res Q Exerc Sport., v. 952, n. 23, p. 115-118.

ZAMBON, T. B. et al. Análise comparativa da flexibilidade de mulheres idosas ativas e não ativas. Acta fisiátr., v. 22, n. 1, p. 14-18, 2016.

ZHANG, X. Y.; SHUAI, J.; LI, L. P. Vision and relevant risk factor interventions for preventing falls among older people: a network meta-analysis. Sci rep., v. 28, n. 5, p. $1-8,2015$.

Recebido em: 17 de setembro de 2016 Aceito em:14 de abril de 2017 